\title{
THEORETICAL AND EXPERIMENTAL STABILITY OF TWO TRANSLATING CABLE EQUILIBRIA
}

\author{
N. C. Perkins \\ Department of Mechanical Engineering and Applied Mechanics, University of Michigan, \\ Ann Arbor, MI 49109, U.S.A. \\ AND \\ C. D. Mote, JR. \\ Department of Mechanical Engineering, University of California, Berkeley, CA 94720, U.S.A.
}

(Received 5 March 1987, and in revised form 25 July 1988)

\begin{abstract}
Previous theoretical investigations of translating cables have been focused on the sagged cable equilibrium referred to here as the minimum catenary. The minimum catenary is stable for finite translation speeds $[1,2]$ and asymptotically approaches a buckling instability as the translation speed tends to infinity [2]. Experimental evidence presented in this paper validates this conclusion and the cable theory established in reference [2]. A second translating cable equilibrium is predicted in which the cable stands in the shape of an arch. This equilibrium, referred to as the maximum catenary, collapses for low translation speeds but becomes stable for sufficiently high translation speeds. The stability of the high speed maximum catenary is confirmed by experiment.
\end{abstract}

\section{INTRODUCTION}

The translating cable represents a technically important variation of the classical translating string originally investigated by Skutch [3] in 1897. The translating string [4], commonly referred to as the moving threadline [5], models the transverse vibration of a taut string having uniform translation speed and passing through two eyelets. The translating cable $[1,2,6]$ models the three-dimensional vibration of a sagged cable having uniform translation speed and passing through two eyelets which may be at different elevations. Unlike the idealized translating string equilibrium which is straight, the translating cable equilibrium sags under the influence of gravity and forms an equilibrium plane. The three-dimensional cable motion is described by components lying in, and normal to, the equilibrium plane.

In 1972, Simpson [1] analyzed the linear in-plane vibration of translating cables having small sag and level eyelets. His analysis reveals that the cable tension increases with translation speed, and the translating cable cannot experience a buckling instability like the (straight) translating string. In 1985, Triantafyllou [6] extended the translating cable problem to include cables having either small or large sag and inclined eyelets. The phenomena of frequency crossover and frequency avoidance greatly influence the dynamic component of cable tension. Recently, Perkins and Mote [2] analyzed both in-plane and out-of-plane linear vibrations of translating cables having arbitrary sag and arbitrary eyelet inclination. They noted that for any non-zero equilibrium sag, the cable asymptotically approaches a buckling instability in the limit of infinite translation speed.

The above analyses focus on a single cable equilibrium which is the familiar catenary of elementary statics modified to incorporate cable translation speed. Here, this equilibrium is referred to as the minimum catenary. There exists a second arch-like equilibrium 
formed by an inverted minimum catenary which is referred to here as the maximum catenary. Without translation speed, the maximum catenary collapses under compressional loading. For sufficiently large translation speed, however, the maximum catenary becomes tensioned and stable [7].

The purpose of this paper is to present experimental evidence in support of the translating cable theory $[2,7]$ and to examine the stability of the minimum catenary and the maximum catenary equilibria by using both experimental and theoretical methods. The cable model of references [2] and [7] is summarized and the experimental apparatus detailed in reference [7] is briefly described.

\section{SUMMARY OF CABLE MODEL}

\subsection{MODELING ASSUMPTIONS}

Figure 1 depicts an elastic sagged cable translating between two fixed eyelets. The two space curves $\chi^{i}$ and $\chi^{f}$ represent the cable equilibrium and final configurations. ${ }^{t}$ The cable equilibrium lies in the vertical $X_{1}-X_{2}$ plane with gravity $\mathrm{g}$ aligned with the $-\mathrm{e}_{2}$ direction. The vector $\mathbf{U}\left(S^{i}, T\right)$ is the three-dimensional motion of the final configuration, and it is distinguished from the motion of a cable particle which includes the particle translation velocity $c^{f} \mathbf{l}_{1}^{f}$. $U\left(S^{i}, T\right)=U_{1} \mathbf{l}_{1}^{i}+U_{2} \mathbf{l}_{2}^{i}+U_{3} \mathbf{l}_{3}^{i}$ is resolved into components aligned with the local tangential $l_{1}^{i}$, normal $l_{2}^{i}$, and bi-normal $l_{3}^{i}$ directions of the cable equilibrium. $S^{i}$ represents the cable equilibrium arc length co-ordinate and $T$ represents time.

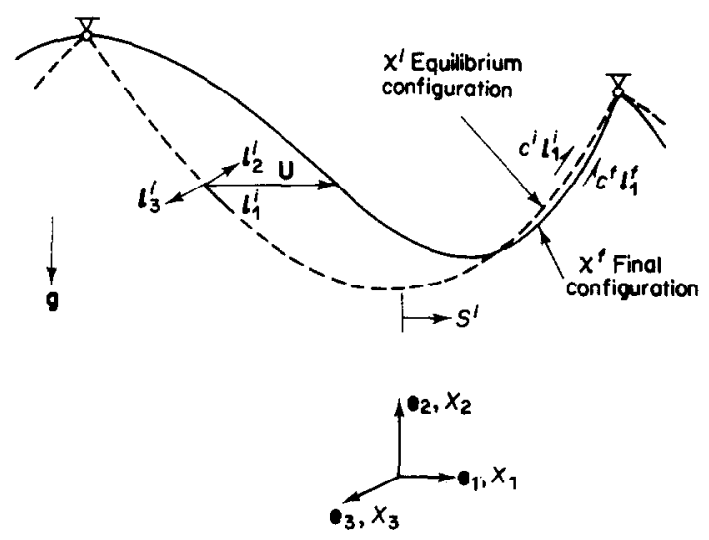

Figure 1. Definition diagram for cable equilibrium and final configurations. Displacement $U$ is referred to Frenet triad $\left(1_{1}^{i}, 1_{2}^{i}, 1_{3}^{i}\right)$. Cable translation velocity relative to $\chi^{i}$ and $\chi^{f}$ is denoted by $c^{i} 1_{1}^{i}$ and $c^{i} 1_{1}^{f}$, respectively.

A derivation of the equations of motion can be found in references $[2,7]$ and is based on the following assumptions: (1) elastic and gravitational forces act on the cable; dissipative forces are not included; (2) the cable is a homogeneous, one-dimensional elastic continuum obeying a linear stress-strain relationship; (3) the cable undergoes uniform axial extension; the axial strains may be large and are described by the Lagrangian strain of the cable centerline; cable bending and torsion are not considered; (4) the cable mass flux $\rho A^{i} c^{i}$ is constant; $\rho, A^{i}$, and $c^{i}$ are the cable mass density, equilibrium cross-sectional area, and equilibrium translation speed, respectively; (5) the cable motion

† Superscripts $i$ and $f$ refer to quantities associated with the cable equilibrium and final configurations, respectively. 
$\mathrm{U}\left(S^{i}, T\right)$ vanishes at the eyelets. Under these assumptions, the non-linear equations of motion are derived by using Hamilton's principle in references $[2,7]$.

\subsection{THE TWO CABLE EQUILIBRIA}

The equations of equilibrium presented in references $[2,7]$ are non-linear in the unknown equilibrium tension $P^{i}\left(S^{i}\right)$ and equilibrium curvature $K^{i}\left(S^{i}\right)$. Introducing the normally very good assumption $\dagger$

$$
P^{i} / E A^{i} \ll 1,
$$

where $E$ is Young's modulus, results in integrable equations of equilibrium given by

$$
\frac{\mathrm{d} P^{i}}{\mathrm{~d} S^{i}}=\frac{\rho g A^{i}\left(\rho g A^{i} S^{i}\right)}{P^{i}-\rho A^{i} c^{i 2}}, \quad K^{i}=\rho g A^{i}\left(\frac{P_{0}-\rho A^{i} c^{i 2}}{\left[P^{i}-\rho A^{i} c^{i 2}\right]^{2}}\right)
$$

The constant $P_{0}$ represents the tension at the extremum point $S^{i}=0$ in Figure 1. Integration yields

$$
P^{i}\left(S^{i}\right)=\rho A^{i} c^{i 2} \pm \rho g A^{i}\left[\left(M^{2}\right)^{2}+\left(S^{i}\right)^{2}\right]^{1 / 2}, \quad K^{i}\left(S^{i}\right)=M^{2} /\left[\left(M^{2}\right)^{2}+\left(S^{i}\right)^{2}\right]
$$

where

$$
M^{2}=\left(P_{0}-\rho A^{i} c^{i 2}\right) / \rho g A^{i}
$$

Two cable equilibria are indicated by the \pm signs in equation (4). Note from equations (4) and (6) that $M^{2}>0$ for the plus sign solution and $M^{2}<0$ for the minus sign solution. Further manipulation of equation (5) $[2,7]$ yields

$$
X_{2}\left(X_{1}\right)=M^{2}\left[\cosh \left(X_{1} / M^{2}\right)\right], \quad S^{i}\left(X_{1}\right)=M^{2}\left[\sinh \left(X_{1} / M^{2}\right)\right],
$$

where $X_{2}(0)$ is chosen to be $M^{2}$.

Equation (7) is the well known catenary of elementary statics with the catenary parameter $\boldsymbol{M}^{2}$ modified to include the translation speed $c^{i}$. An alternative derivation of equation (6) is given in reference [1] where $M^{2}>0$ is assumed. The equilibria $M^{2}>0$ and $M^{2}<0$ are referred to as the minimum and the maximum catenary, respectively. The terms minimum and maximum denote the extremum values of the gravitational potential energy functional for an inextensible cable of specified length [8]. The functional becomes a minimum at the minimum catenary, and a maximum at the maximum catenary. Both equilibria are illustrated in Figure 2 for the case $\left|M^{2}\right|=0.2988$ and $V / H=1 / 5$, where $H$ and $V$ are the horizontal and vertical distances between the eyelets and $L^{i}$ is the equilibrium cable length.

\subsection{LINEAR EQUATIONS OF MOTION AND DISCRETE MODEL}

The equations of motion are linearized about the cable equilibria in references $[2,7]$, where the following non-dimensional quantities are introduced:

$$
s=\left(S^{i}+L^{*}\right) / L^{i}
$$

where $L^{*}$ is the length of the equilibrium cable from the left eyelet to the extremum point;

$$
t=T\left(g / L^{i}\right)^{1 / 2} ; \quad u_{j}=U_{j} / L^{i} \quad j=1,2,3 ; \quad p=P^{i} / P_{0} ; \quad k=K^{i} L^{i}
$$

The three linearized equations of motion governing free response about the cable equilibria are, for the tangential component, $u_{1}$,

$$
\left[\left(v_{l}^{2}-v_{c}^{2}\right)\left(u_{1, s}-k u_{2}\right)\right]_{, s}-k\left[\left(v_{1}^{2} p-v_{c}^{2}\right)\left(u_{2, s}+k u_{1}\right)\right]=u_{1, t}+2 v_{c}\left(u_{1, s}-k u_{2}\right)_{, r}
$$

$\dagger$ This assumption is equivalent to approximating the cable equilibrium by that of an inextensible cable. 

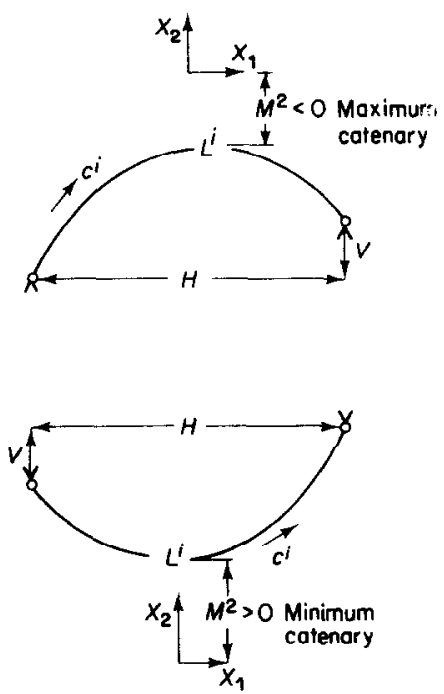

Figure 2. The minimum catenary $\left(M^{2}>0\right)$ and the maximum catcnary $\left(M^{2}<0\right)$ equilibria for the case $\left|M^{2}\right|=0.2988$ and $V / H=1 / 5 . L^{i}$ is the equilibrium cable length and $c^{i}$ is the equilibrium translation speed. $X_{1}=0$ is aligned with the extremum point of the cable equilibrium.

for the normal component, $u_{2}$,

$$
\left[\left(v_{t}^{2} p-v_{c}^{2}\right)\left(u_{2, s}+k u_{1}\right)\right]_{, s}+k\left[\left(v_{l}^{2}-v_{c}^{2}\right)\left(u_{1, s}-k u_{2}\right)\right]=u_{2, t}+2 v_{c}\left(u_{2, s}+k u_{1}\right)_{, t}
$$

and for the bi-normal component, $u_{3}$,

$$
\left[\left(v_{t}^{2} p-v_{c}^{2}\right)\left(u_{3, s}\right)\right]_{, s}=u_{3, t r}+2 v_{c}\left(u_{3, s}\right)_{, t}
$$

and the boundary conditions are $u_{j}(0, t)=u_{j}(1, t)=0$ for $j=1,2,3$.

The three constant coefficients appearing in equations (14), (15), and (16) are characteristic wave speeds given by

$$
\begin{array}{ll}
v_{t}^{2}=P_{0} /\left(\rho g A^{i} L^{i}\right), & \text { transverse wave speed; } \\
v_{l}^{2}=E A^{i} /\left(\rho g A^{i} L^{i}\right) & \text { longitudinal wave speed, } \\
v_{c}^{2}=\left(c^{i}\right)^{2} /\left(g L^{i}\right), & \text { cable transition speed. }
\end{array}
$$

The remaining two coefficients are non-constant and represent the equilibrium curvature and tension distributions:

$$
v_{t}^{2} p(s)=v_{c}^{2} \pm\left[\left(m^{2}\right)^{2}+\left(s-l^{*}\right)^{2}\right]^{1 / 2}, \quad k(s)=m^{2} /\left[\left(m^{2}\right)^{2}+\left(s-l^{*}\right)^{2}\right]
$$

where

$$
m^{2}=M^{2} / L^{i}=v_{t}^{2}-v_{c}^{2} \quad \text { and } \quad l^{*}=L^{*} / L^{i}
$$

For the minimum catenary, the plus sign is chosen in equation (20) and $m^{2}>0$ in equations (21) and (22), and for the maximum catenary, the minus sign is chosen in equation (20) and $m^{2}<0$ in equations (21) and (22).

Inspection of equations (14), (15) and (16) reveals two important features of linear cable motion. First, the linear in-plane motion described by the $u_{1}$ and $u_{2}$ components of equations (14) and (15) decouples from the linear out-of-plane motion described by the $u_{3}$ component of equation (16). Second, the coupling of the in-plane components derives from the equilibrium curvature $k(s)$. Thus, for a straight translating cable $(k=0)$, all three equations decouple and reduce to the equations of motion of the translating string $[3-5]$ and the translating rod. 
General analytical solutions have not yet been found for the complete linear equations of motion which remain coupled and have non-constant coefficients of irrational form. Asymptotic closed-form solutions have been derived for special cases of the minimum catenary $[1,6]$. Here, numerical solutions are computed after discretizing, using the Galerkin method $[2,7]$. Consider $N$-term separable series solution representations for the $u_{i}$ of the form

$$
u_{i}(s, t)=\sum_{j=1}^{N} \phi_{i j}(t) \theta_{j}(s), \quad i=1,2,3
$$

where

$$
\theta_{j}(s)=2^{1 / 2} \sin (j \pi s) .
$$

The discretization detailed in references $[2,7]$ results in the $4 N \times 4 N$ eigenvalue problem

$$
\mathbf{A}\left(\begin{array}{c}
\vec{\phi}_{1} \\
\vec{\phi}_{2}
\end{array}\right)=\omega\left(\begin{array}{c}
\vec{\phi}_{1} \\
\vec{\phi}_{2}
\end{array}\right)
$$

governing in-plane vibration and a $2 N \times 2 N$ eigenvalue problem

$$
\mathbf{B} \vec{\phi}_{3}=\omega \vec{\phi}_{3},
$$

governing out-of-plane vibration. $\operatorname{Im}(\omega)$ and $\operatorname{Re}(\omega)$ represent the cable natural frequencies and damping factors for in-plane and out-of-plane modes determined by using equation (23) with the eigenvectors

$$
\vec{\phi}_{i}=\left(\begin{array}{c}
\phi_{i 1} \\
\phi_{i 2} \\
\vdots \\
\phi_{i N}
\end{array}\right), \quad i=1,2,3
$$

\section{EXPERIMENTAL APPARATUS}

The cable natural frequencies and stability are experimentally determined using the test stand illustrated in Figure 3. The cable (a), having the indicated material properties,

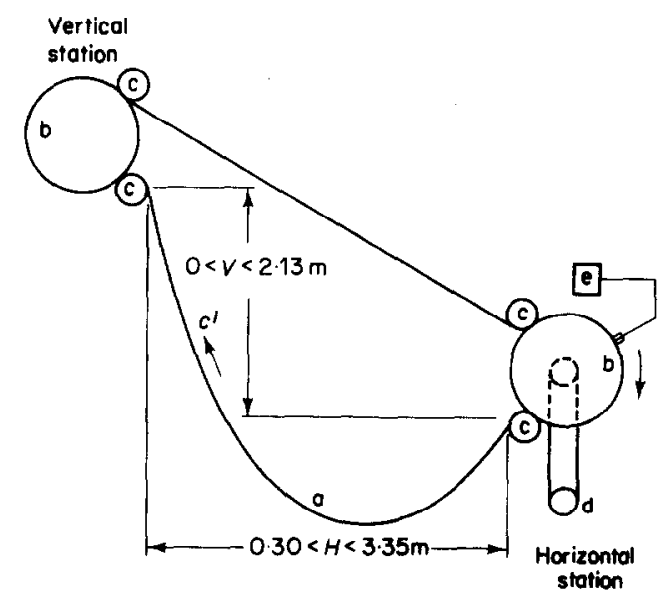

Figure 3. Schematic diagram of the cable test stand. (a) Cable, $4.8 \mathrm{~mm}$ diameter woven nylon rope having weight/length $\rho A^{i} g=6.9 \mathrm{~g} / \mathrm{m}$ and section stiffness $E A^{i}=2200 \mathrm{~N}$; (b) pulley, $30.5 \mathrm{~cm}$ diameter with $4.8 \mathrm{~mm}$ semicircccular groove around perimeter; (c) pinch roller, $8.89 \mathrm{~cm}$ diameter flanged roller attached to an air piston pressurized to $68.9 \mathrm{kPa}$; (d) DC motor, $1 / 2 \mathrm{hp}$ Emerson TR-50 equipped with speed control and forward/reverse switch; (e) translation speed, magnetic pick-up and Beckman 6240 frequency counter. 
translates between the horizontal and vertical stations separated by the distances $H$ and $V$. The cable wraps around a pulley (b) at each station. Surgical tubing rubber lines a groove in the pulley perimeter and increases pulley/cable friction. Pinch rollers (c), drawn radially inwards against the cable by pressurized air pistons, guide the cable on and off the pulleys. A DC motor (d) drives the pulley on the horizontal station in the clockwise direction creating a taut (top) span and a sagged (lower) span. Experiments were focused on the sagged span which achieves translation speeds $c^{i}$ up to $20 \mathrm{~m} / \mathrm{s}$, as measured by a pick-up and frequency counter (e).

Figure 4 is a schematic diagram of a two-axis optical displacement probe developed to measure cable motions at one point along, and perpendicular to, the cable centerline. The probe simultaneously measures the out-of-plane motion component, $U_{3}$, and the normal component of the in-plane motion, $U_{2}$. Along each axis, a fluorescent light (a) illuminates the cable (b) which casts a focused (c) shadow on a diode array (d). The array contains 256 diodes on $25 \mu \mathrm{m}$ centers. A sampling circuit (e) starts a counter and simultaneously begins to scan the diode array. When a diode output falls below a prescribed threshold value, the counter is read providing the analog voltage signal proportional to $U_{2}$ (or $U_{3}$ ). Scanning rates up to $3900 \mathrm{~Hz}$ easily capture the $0-10 \mathrm{~Hz}$ cable motions which are of primary interest, and a $100 \mathrm{~Hz}$ low-pass filter reduces measurement noise. Cable motions within a $6.0 \mathrm{~cm}$ by $6.0 \mathrm{~cm}$ sensing area are resolved to within an error of $1 \mathrm{~mm}$ [7].

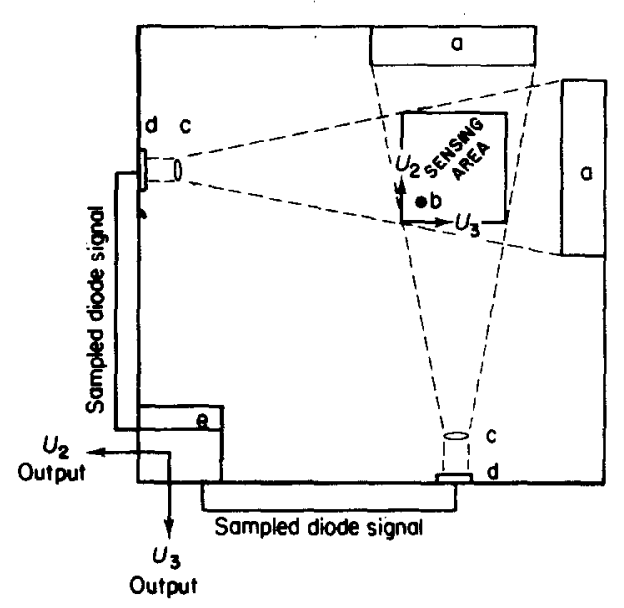

Figure 4. Schematic of two-axis displacement probe. Sensing area is $6 \mathrm{~cm}$ by $6 \mathrm{~cm}$. (a) Fluorescent light, $25 \mathrm{~cm}, 10 \mathrm{~W}$; (b) cable, see (a) Figure 3; (c) lens, $12.7 \mathrm{~mm}$ diameter focusing lens having a $9.5 \mathrm{~mm}$ focal length; (d) diode array, Reticon RL256G diode array having 256 diodes on $25 \mu \mathrm{m}$ centers; (e) sampling circuit, Reticon RC301 PC board recharge amplifier and a sample-and-hold circuit.

The in-plane and out-of-plane cable natural frequencies are measured by using an HP 5423A Structural Dynamics Analyzer. The two-channel analyzer computes the autocorrelation functions for free in-plane and out-of-plane $\left(U_{2}\right.$ and $\left.U_{3}\right)$ response following initial in-plane and out-of-plane disturbances. Figure 5 shows a sample autocorrelation function for the out-of-plane response of the illustrated stationary cable. The highest peak marks the location of the fundamental cable frequency, $0.76 \mathrm{~Hz}$. The peaks associated with higher cable modes decrease rapidly with mode number and become broader and poorly defined. The same pattern holds for the autocorrelation function for the in-plane co-ordinate $\mathrm{U}_{2}$. Large internal cable damping derives from the woven construction of the nylon rope and quickly limits the contribution of higher cable modes to the response. 


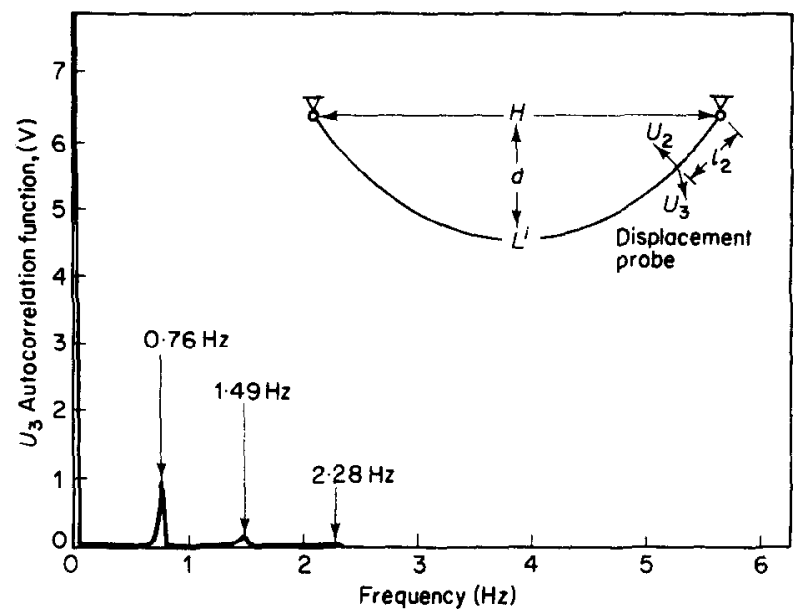

Figure 5. Sample autocorrelation function for free out-of-plane $U_{3}$ response. The first three out-of-plane natural frequencies are indicated by the locations of the peaks. $L^{i}=2.44 \mathrm{~m}, H=1.99 \mathrm{~m}$ and $d=0.61 \mathrm{~m}$. The probe is positioned at $l_{2}=0.64 \mathrm{~m}$ from the right eyelet.

The second and third out-of-plane natural frequencies are barely discernible in Figure 5 , and an expanded scale function is used to locate them properly. An eyelet attached to a voice coil was added in an attempt to drive higher cable modes [7], but large internal cable damping again prevented the excitation of cable modes beyond the third.

\section{VALIDATION OF CABLE MODEL}

The in-plane and out-of-plane frequency spectra predicted by the discretized cable model are compared with experimentally determined values for the translating minimum catenary. A 3.47 m length of cable is suspended between the cable stations separated by $H=2.23 \mathrm{~m}$ and $V=0.91 \mathrm{~m}$ as illustrated in Figure 6. The in-plane and out-of-plane frequencies are measured as a function of translation speed. Figures 6 and 7 show the first three in-plane and the first three out-of-plane frequencies measured at 12 translation

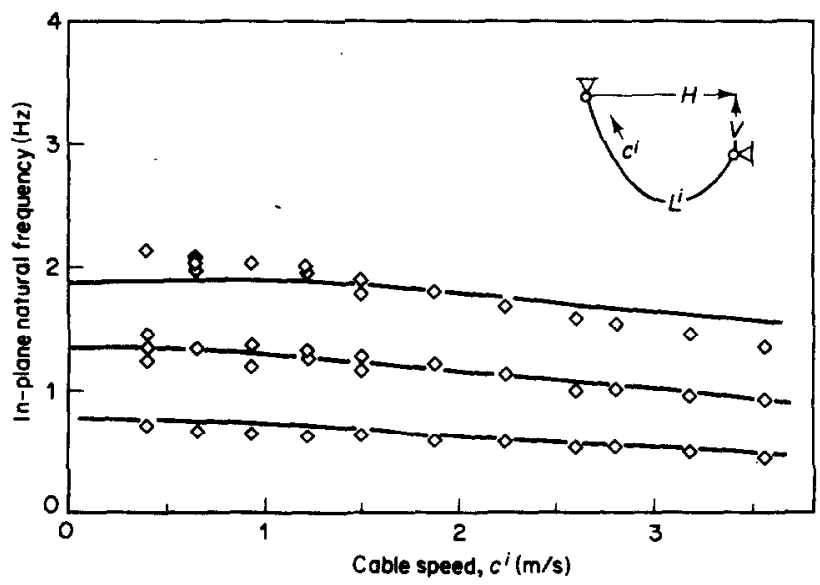

Figure 6. Experimental and theoretical in-plane natural frequencies for a low speed translating cable having inclined eyelets. $L^{i}=3.47 \mathrm{~m}, H=2.23 \mathrm{~m}$, and $V=0.91 \mathrm{~m} . N=10$ in equation (23). _-, Cable model; $\diamond \diamond \diamond$, experiment. 


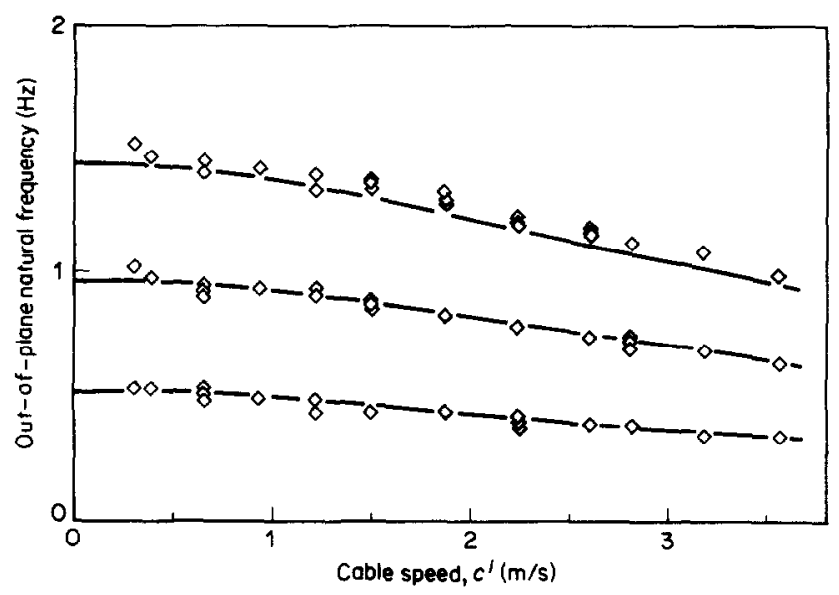

Figure 7. Experimental and theoretical out-of-plane natural frequencies for a low speed translating cable having inclined eyelets. Cable equilibrium is described in Figure 6. Key as Figure 6.

speeds ranging from 0 to $3.66 \mathrm{~m} / \mathrm{s}$. The close and vertically spaced data points in these figures represent multiple frequency measurements, and the solid curves represent the frequencies calculated from the cable model. Inspection of Figures 6 and 7 reveals excellent agreement between measured and computed cable natural frequencies which gradually decrease over the indicated speed range.

\section{STABILITY OF THE MINIMUM CATENARY}

A translating string having tension $P_{0}$ and translation speed $c^{i}$ experiences a buckling instability when

$$
v_{c}^{2} / v_{t}^{2}=1,
$$

where the non-dimensionalization of section 2.3 is used $[4,5]$. For a translating cable of constant length, the catenary parameter $m^{2}=v_{1}^{2}-v_{c}^{2}$ of equations $(22)$ is constant $[2,7]$ and relates the cable tension $v_{t}^{2}$ to the translation speed $v_{c}^{2}$. The above buckling condition becomes

$$
v_{c}^{2} /\left(v_{c}^{2}+m^{2}\right)=1
$$

and is satisfied in the limit $v_{c}^{2} \rightarrow \infty$. The cable tension must increase in response to an increase in translation speed in order to accelerate a cable particle along the constant and curved cable equilibrium. This prediction, first made by Simpson [1], has been tested experimentally, as follows.

Figure 8 depicts a $1.58 \mathrm{~m}$ length of cable suspended between the stations separated by $H=1.16 \mathrm{~m}$. The fundamental in-plane and out-of-plane frequencies were measured at 12 translation speeds ranging from 0 to $13.4 \mathrm{~m} / \mathrm{s}$. In this test, exceptionally large cable damping prevents measurable excitation of the second and higher cable modes. Large aerodynamic damping forces are generated at high translation speeds and supplement the already large internal damping. The theoretical predictions (solid curves) are shown together with the experimental data. The fundamental frequencies rapidly decrease with translation speed and at $c^{i}=13.4 \mathrm{~m} / \mathrm{s}$ they are less than $1 / 7$ of their values at $c^{i}=0$. The cable approaches a buckling instability as the fundamental frequencies asymptotically 


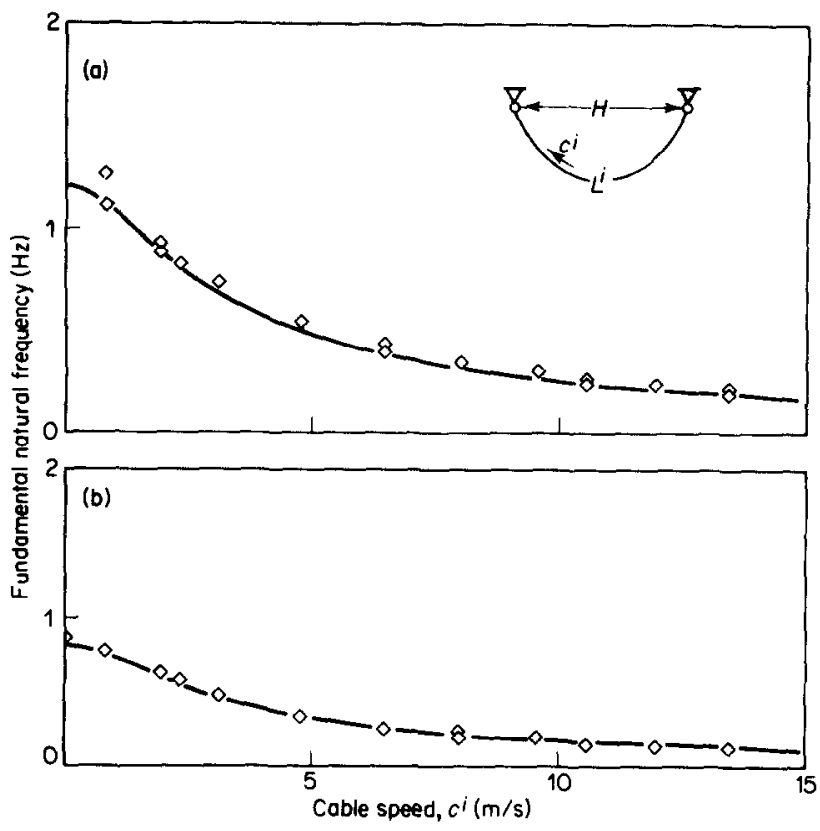

Figure 8. Experimental and theoretical fundamental frequencies for a high-speed transiating minimum catenary. (a) In-plane; (b) out-of-plane. $L^{i}=1.58 \mathrm{~m}$ and $H=1.16 \mathrm{~m} . N=10$ in equation (23). Key as Figure 6.

approach zero with increasing translation speed. The cable model predicts the same trend for higher order frequencies; see Figure 9 of reference [2].

The asymptotic behavior of the fundamental frequencies, as just described, is reflected by the propagation speeds of disturbances traveling along the cable. An initial transverse cable displacement creates two d'Alembert waves: (1) a forward traveling wave propagating in the direction of cable translation, and (2) a backward traveling wave propagating in the opposite direction. The propagation speeds of the out-of-plane d'Alembert waves are readily computed from the characteristics associated with the linear hyperbolic partial differential equation of motion (16). The two (real) characteristics in the $t-s$ plane satisfy [9]

$$
\frac{\mathrm{d} t}{\mathrm{~d} s}=\frac{-v_{\mathrm{c}} / m \pm\left\{\left(v_{\mathrm{c}} / m\right)^{2}+\left[1+\left(s-l^{*} / m^{2}\right)^{2}\right]^{1 / 2}\right\}^{1 / 2}}{m\left[1+\left(s-l^{*} / m^{2}\right)^{2}\right]^{1 / 2}} .
$$

The propagation speeds are given by $\mathrm{d} s / \mathrm{d} t$ and the \pm signs in equation (30) designate the forward and backward traveling waves, respectively. At $v_{c} / m=0$, the propagation speeds of the forward and backward traveling waves are equal in magnitude, of opposite sign, and vary with $s$ due to the non-uniform tension along the stationary sagged cable. Translation speed destroys the symmetry of the stationary cable solution, and for $v_{c} / m>0$ the forward traveling wave propagates faster than its backward traveling counterpart. A dramatic difference in the propagation speeds of the forward and backward traveling waves is observed at high translation speed. At $c^{i}=13.4 \mathrm{~m} / \mathrm{s}$ for the cable of Figure 8 , an initial mid-span disturbance creates a forward traveling wave which reflects from the downstream (left) station almost instantaneously. By contrast, the backward traveling wave propagates so slowly that it often never reaches the upstream (right) station, which is only $0.79 \mathrm{~m}$ away, before being dissipated by damping forces. In the limit $v_{c} / m \rightarrow \infty$ in equation (30), the propagation speed of the backward traveling wave vanishes and standing wave resonance leads to the buckling instability discussed above. 


\section{STABILITY OF THE MAXIMUM CATENARY}

Without translation speed, the maximum catenary collapses under compressional loading. With translation speed however, the cable can be in tension as seen by the minus sign solution of equation (4). The entire cable is in tension for speeds $v_{c} / m>$ $\left[1+\left(1 / 4 m^{4}\right)\right]^{1 / 4}$ and is in tension in the interval $|s-1 / 2|<m^{2}\left[\left(v_{c}^{2} / m^{2}\right)^{2}-1\right]^{1 / 2}$ for speeds $1<v_{c} / m<\left[1+\left(1 / 4 m^{4}\right)\right]^{1 / 4}$, where $m$ denotes IM $[m]$. This speed tensioning effect allows the initially unstable equilibrium to become stable at sufficiently high translation speeds. Stable vibration modes are associated with eigenvalues having non-positive real parts, as determined from the discrete eigenvalue problems (25) and (26).

The eigenvalues governing the stability of the first eight out-of-plane modes are shown in Figure 9 as functions of translation speed $v_{c} / m$. In this example, $m^{2}=-0 \cdot 2988$, and the maximum catenary has the illustrated shape. The real eigenvalues below $v_{c} / m=1$ become imaginary above $v_{c} / m=1$ and indicate stability for the out-of-plane vibration modes. Stability occurs as the eigenvalues pass through a singularity at $v_{c} / m=1 ; \operatorname{Re}[\omega] \rightarrow$ $\infty$ from below and $\operatorname{Im}[\omega] \rightarrow \infty$ from above. To ensure eigenvalue convergence, an increasingly larger expansion size in equation $N(23)$ is required as $v_{c} / m \rightarrow 1$. Note that Figure 9 shows the first eight eigenvalues only in the regions where they have converged for the case where $N=70$. For this large value of $N$, the fundamental eigenvalue has converged everywhere except in the rather small interval $0.99 \leqslant v_{c} / m \leqslant 1.04$. An analytical solution for the eigenvalues of the continuous problem (16) has recently been developed which confirms the singularity approximated in Figure 9. The analytical solution is valid for shallow maximum catenaries and is the subject of a subsequent publication.

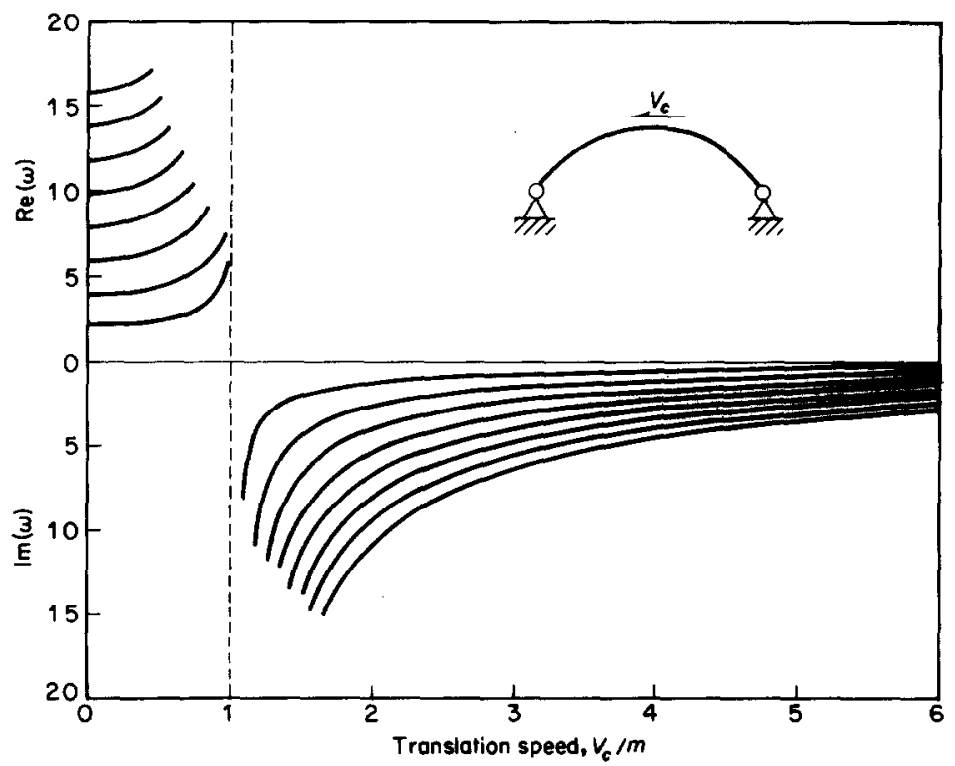

Figure 9. Stability of out-of-plane modes for a translating maximum catenary. Real and imaginary parts of eigenvalues are plotted versus translation speed for the case $m^{2}=-0.2988$ and $N=70$ in equation (23). Stability is determined by $\operatorname{Re}\left(\omega_{n}\right) \leqslant 0$.

The eigenvalues for the first eight in-plane modes are plotted versus translation speed in Figure 10 for the same maximum catenary shown in Figure 9. In contrast to the out-of-plane problem, the eigenvalues for the in-plane modes become complex during 


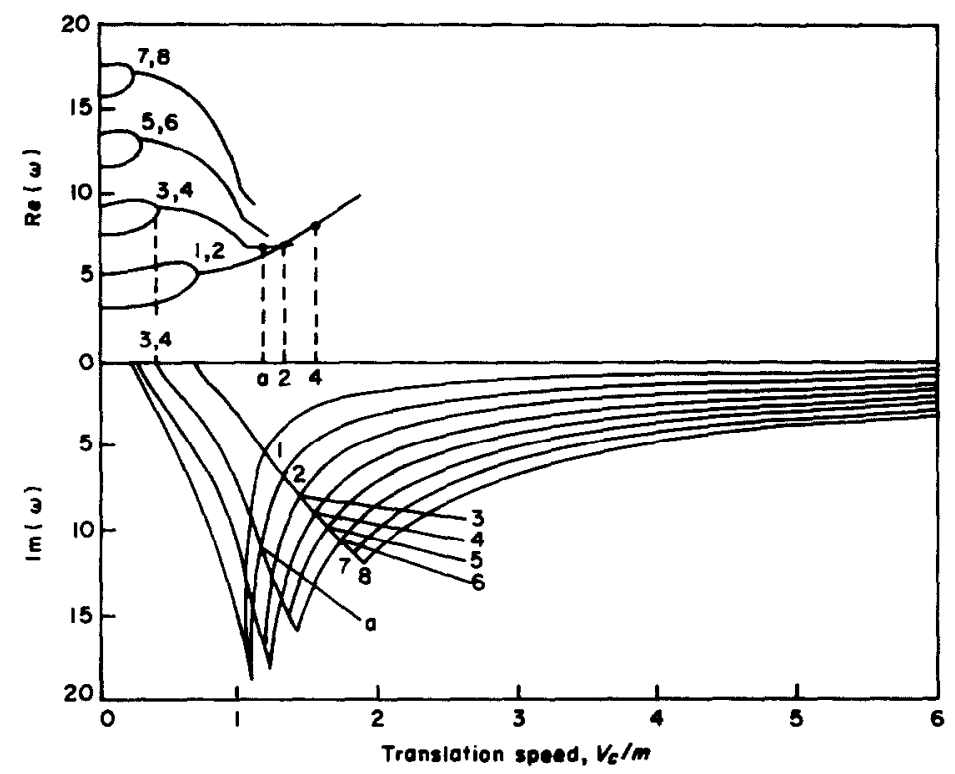

Figure 10. Stability of in-plane modes for a translating maximum catenary. Real and imaginary parts of eigenvalues are plotted versus translation speed for the case $m^{2}=-0.2988, v=5000$ and $N=70$ in equation (23). Cable equilibrium is shown in Figure 9.

the transition from real to imaginary values. All the eigenvalues are real at $v_{c} / m=0$ and remain real until the adjacent pairs of real loci coalesce and a common imaginary locus is formed. This transition from real to complex eigenvalues, which occurs simultaneously for the $n$th and $(n+1)$ st eigenvalues, is marked by the $n, n+1$ in the upper portion of Figure 10. Following this transition, the complex eigenvalues are described by $\omega_{r}=$ $\pm \operatorname{Re}\left(\omega_{r}\right) \pm \mathrm{i} \operatorname{Im}\left(\omega_{r}\right)$, for $r=n, n+1$, and indicate instability for the $n$th and $(n+1)$ th in-plane modes. The eigenvalue for the $n$th in-plane mode becomes imaginary following a second transition marked by $n$ in the lower portion of Figure 10, and the $n$th mode remains stable for translation speeds beyond this point. Between the points $n, n+1$ and $n$, the $n$th eigenvalue alternates between complex and pure imaginary values. As an example, the behavior of the fourth eigenvalue can be understood by reference to the points marked 3,4, a, 2, and 4 in Figure 10. At 3,4 the (real) third and fourth eigenvalues coalesce and a common imaginary locus appears. The fourth eigenvalue remains complex between points 3,4 and a, becomes imaginary in the short interval between a and 2 , is again complex between points 2 and 4 , and remains imaginary beyond 4 . A recently developed analytical solution for the in-plane shallow maximum catenary problem confirms the transition of the eigenvalues from real to complex to imaginary values observed in Figure 10. This analytical solution will also be included in the subsequent publication referred to above.

Figure 10 shows that the translation speed required to stabilize in-plane modes increases with mode number, and at any translation speed there always exist unstable high-order in-plane modes. It is unlikely, however, that such high-order modes will contribute to the response of the highly damped cable considered in the experiments. In reference [7], linear damping terms are appended to the equations of motion in an attempt to model cable dissipation. With damping added, all $\operatorname{Re}\left(\omega_{n}\right)$ loci approach the same limiting negative value but the stabilization speeds of Figure 10 are virtually unchanged. While the simple linear damping model adds stability to all modes, it does not preferentially 
stabilize high-order modes first. More accurate modeling of the large and complicated dissipative forces may alter this result.

The stability of the maximum catenary has been confirmed by experiment. For a maximum catenary having $m^{2} \approx-0.2988$ and $L^{i}=1.02 \mathrm{~m}$, the test stand can achieve translation speeds up to $v_{c} / m=11.6$ which is well above the stabilization speeds for low-order modes predicted in Figures 9 and 10. An equilibrium having $m^{2} \approx-0.2988$ is obtained by adjusting $H$ until an arch height to span ratio of 0.37 is achieved. The photograph in Figure 11 shows the cable being supported at the mid-span point while the cable is stationary. Plexiglass guides are positioned above the pinch rollers to guide the rope as it enters on and off the pulleys. The rope easily derails from the pulleys at high translation speed if left unguided. The cable of Figure 11 is supported while the translation speed is increased, and when $c^{i}=13.8 \mathrm{~m} / \mathrm{s}\left(v_{c} / m=8\right)$, the cable is released and stands upright. Figure 12 illustrates the stable translating maximum catenary equilibrium.

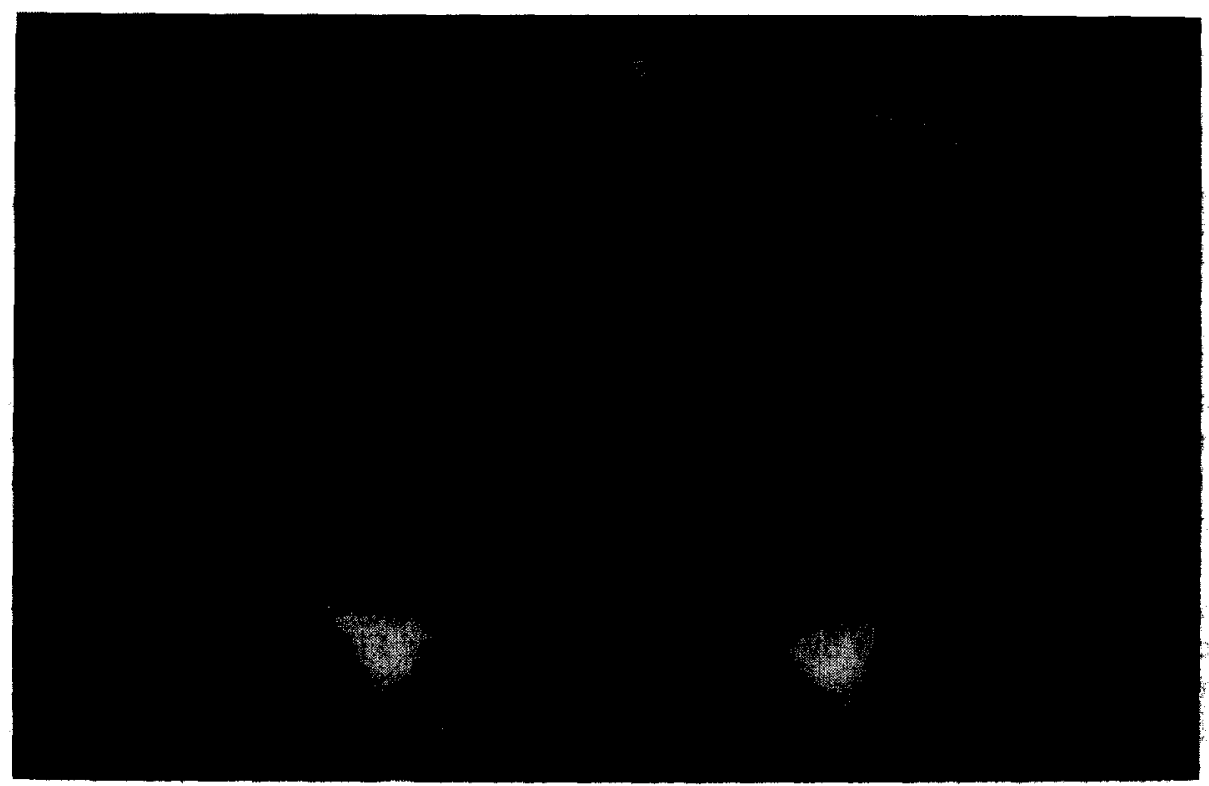

Figure 11. Maximum catenary before stabilization. Nylon rope of length $L^{\prime}=1.02 \mathrm{~m}$ is supported at mid span point when $c^{i}=0$. Note the Plexiglass guides at the $2 o^{\prime}$ 'clock position on the right pulley and the $10 o^{\prime}$ clock position on the left pulley.

Three important qualitative observations are noted presently. First, the translating maximum catenary at $v_{c} / m=8 \cdot 0$ remains surprisingly quiescent for the duration of the 10 minute test. The cable maintains its equilibrium shape despite being subjected to small but continuous disturbing forces that arise from drive train noise, pulley eccentricities and air currents. Furthermore, the cable recovers its equilibrium shape following initial in-plane and out-of-plane displacements as large as $2-3 \mathrm{~cm}$ applied near the pulleys and at the mid-span point. Second, when the translation speed is reduced to $v_{c} / m=6.0$ $(10.4 \mathrm{~m} / \mathrm{s})$ large and slow oscillations eventually drive the cable out of the equilibrium plane at the downstream (left) pulley. The maximum catenary equilibrium could not be maintained below $v_{c} / m=6.0$. Third, the transition to instability is gradual. Between $v_{c} / m=8.0$ and 6.0 the stability of the translating cable is not well defined. In this speed range, the cable often recovers from $2-3 \mathrm{~cm}$ initial displacements applied at the mid-span 


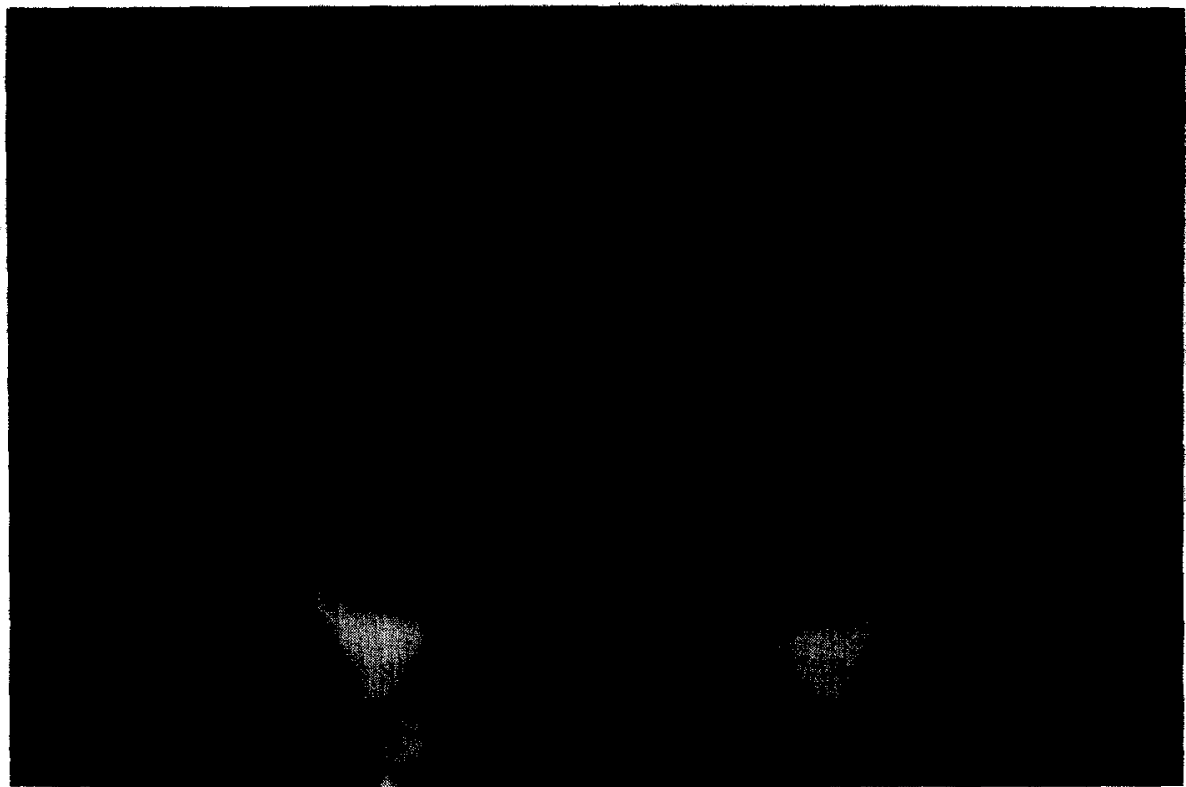

Figure 12. Maximum catenary after stabilization. Photograph captures the stable translating maximum catenary equilibrium. Cable circulates counterclockwise. $L^{i}=1 \cdot 02, m^{2}=-0.2988$ and $c^{i}=13.8 \mathrm{~m} / \mathrm{s}\left(v_{c} / m=8 \cdot 0\right)$.

point but frequently fails to recover from initial displacements applied near the downstream pulley.

\section{SUMMARY AND CONCLUSIONS}

The equations of cable equilibrium admit two solutions referred to as the minimum catenary and the maximum catenary. The cable model predicts that the minimum catenary remains stable for finite translation speeds but asymptotically approaches a buckling instability as the translation speed tends to infinity. The absence of a finite critical translation speed is attributed to the speed-dependent cable tension. The cable stability has been demonstrated by measuring the fundamental in-plane and out-of-plane frequencies at high translation speeds, and the measured frequencies agree with those predicted by the cable model.

The initially unstable maximum catenary can be stabilized by the addition of cable translation speed. At high translation speeds, the cable is tensioned and the theoretical model predicts cable stability. This result also has been confirmed by experiment and by an analytical solution to the shallow maximum catenary problem, detailed in a forthcoming publication.

\section{ACKNOWLEDGMENTS}

The authors express their gratitude to Professor P. Hagedorn of the Institute für Mechanik II-Darmstadt, who suggested an investigation of the maximum catenary and to the National Science Foundation and the Exxon Education Foundation for their partial support of this research endeavor. The authors also gratefully acknowledge a reviewer's advice on the convergence behavior of the maximum catenary out-of-plane frequencies. 


\section{REFERENCES}

1. A. SIMPSON 1972 Jourmal of Sound and Vibration 20(2), 177-189. On the oscillatory motions of translating elastic cables.

2. N. C. PERKINS and C. D. MOTE, JR. 1987 Journal of Sound and Vibration 114, 325-340. Three dimensional vibration of travelling elastic cables.

3. R. SKUTCH 1897 J. Ann. Phys. Chem. 61, 190-195. Über die Bewegung eines gespannten Fadens, welcher gezwungen ist, durch zwei feste Punkte, mit einer constanten Geschwindigkeit zu gehen, und zwischen denselben in Transversal-Schwingungen von geringer Amplitude versetzt wird.

4. R. A. SACK 1954 British Journal of Applied Physics 5, 224-226. Transverse oscillations in travelling strings.

5. R. D. SwOPE and W. F. AMES 1963 Journal of the Franklin Institute 275(1), 36-55. Vibrations of a moving threadline.

6. M. S. TRIANTAFYlLou 1985 Journal of Sound and Vibration 103, 171-182. The dynamics of translating cables.

7. N. C. Perkins 1986 Ph.D. Dissertation, University of California, Berkeley. Three dimensional theory and analysis of travelling sagged cable dynamics.

8. R. CourANT and D. Hilbert 1953 Methods of Mathematical Physics, Vol. 1. New York: John Wiley. See chapter 4.

9. C. R. WYLIE and L. C. BARRETT 1982 Advanced Engineering Mathematics (fifth edition). New York: McGraw-Hill. 